\title{
Trabajo autónomo y en equipo potenciados vía prácticas de aula
}

\author{
Francisco J. Suárez, Juan C. Granda, Víctor Corcoba \\ Universidad de Oviedo, Departamento de Informática, Gijón, España, \\ \{fjsuarez, jcgranda, corcobavictor\}@uniovi.es
}

\begin{abstract}
Resumen
Nuestra propuesta trata de promover el trabajo autónomo del alumnado en una materia de un título universitario en ingeniería como vía para mejorar el rendimiento del trabajo en equipo. Tanto los contenidos como la evaluación de las prácticas de aula en la asignatura se han rediseñado, habiendo logrado resultados significativos no solo en el rendimiento, sino también en la asistencia a las prácticas de aula y la satisfacción del alumnado.
\end{abstract}

Palabras clave: trabajo autónomo, trabajo en en equipo, prácticas de aula

\section{Introducción}

El proyecto surge como respuesta a dos aspectos de la práctica docente con margen de mejora en la asignatura Servicios Multimedia e Interactivos, de tercer curso del Grado en Ingeniería en Tecnologías y Servicios de Telecomunicación, impartida en la Escuela Politécnicas de Ingeniería de Gijón de la Universidad de Oviedo. Por una parte, el trabajo en equipo de la asignatura implica una disciplina de autoaprendizaje mediante trabajo autónomo que no todos los miembros del equipo llevan a cabo de forma organizada, lo que repercute en los mediocres resultados de algunos equipos. Por otra parte, se observa una caída sostenida de la asistencia a las prácticas de aula de la asignatura a medida que avanza el curso, cayendo rápidamente por debajo del 50\%. La razón de la baja asistencia se estima debida a que buena parte de las prácticas de aula no aportan contenidos evaluables en la asignatura. Con el presente proyecto se trata de paliar estos dos problemas en base al rediseño tanto de los contenidos como de la evaluación de las prácticas de aula, buscando mejorar tanto el rendimiento del trabajo en equipo como la asistencia a las clases y como consecuencia también la satisfacción del alumnado. El proyecto parte de la experiencia en un proyecto de innovación anterior en la misma asignatura donde se planteaban objetivos similares en cuanto a asistencia a clases expositivas y mejora de la cooperación en los trabajos equipo mediante herramientas colaborativas (Suárez, 2018). 


\section{Objetivos}

1. Potenciar el trabajo autónomo del alumno.

2. Mejorar del rendimiento del trabajo en equipo.

3. Mejorar la asistencia a prácticas de aula.

4. Mejorar la satisfacción del alumno.

\section{Marco teórico}

El proyecto propuesto parte de los fundamentos del aprendizaje basado en problemas y su puesta en práctica a través de trabajos en equipo.

En el Aprendizaje Basado en Problemas (ABP) los alumnos toman responsabilidades y acciones que son básicas en su proceso formativo, y por ello se considera que esta forma de trabajo representa una alternativa necesaria en la formación de profesionales, especialmente en el caso de la ingeniería. Resulta además un método factible para ser utilizado por los profesores, con mayor o menor intensidad, en la mayor parte de las disciplinas (Monterrey, 2008).

El trabajo en equipo aporta importantes competencias al alumnado en la educación superior en general (Burke, 2011) y en la ingeniería en particular (Marín-García, 2008; Lingard, 2011). La duración de las actividades colaborativas a evaluar es un aspecto de suma importancia, ya que condiciona en gran medida las interacciones entre los estudiantes (Viswanathan, 2017). Gran parte de los estudios que podemos encontrar en la literatura corresponden a actividades colaborativas en el ámbito de una sesión docente, es decir, en torno a una hora. En todo caso, existen también algunos estudios sobre equipos de estudiantes que trabajan juntos durante varias semanas o un semestre completo utilizando herramientas tales como foros (Anaya, 2011), chats o wikis y repositorios de código (Perera, 2009). Este tipo de trabajos es sin duda mucho más acorde al planteado en nuestro proyecto de innovación, ya que vamos a considerar trabajos en equipo de 8 semanas de duración.

Por otra parte, el tipo de competencias transversales y técnicas requeridas por el alumno para el eficaz desarrollo de un trabajo en equipo no siempre son proporcionadas por las actividades planteadas en las asignaturas, lo cual repercute en un bajo rendimiento de algunos equipos. Las prácticas de aula podrían ser una buena vía para cubrir ese déficit de competencias. Las prácticas de aula constituyen un tipo de docencia en la que el profesor ilustra con casos de estudio o resoluciones prácticas a un grupo reducido de estudiantes. 
Aunque el profesor interacciona con los estudiantes, es el profesor es el que lleva el peso de la clase. Es un tipo de docencia que complementa con aspectos prácticos la teoría expuesta en las clases magistrales y que resulta idónea para proponer diferentes tareas semanales a modo de trabajo autónomo, coordinar los diferentes equipos de trabajo de la asignatura y transmitir pautas comunes sobre la forma de llevar a cabo los trabajos.

Existen precedentes del uso de las prácticas de aula para trabajar las competencias transversales con buenos resultados (Ardid, 2014). En este proyecto de innovación se consideran tanto las competencias transversales como las competencias técnicas necesarias para el desarrollo del trabajo en equipo de la asignatura. Ambos tipos de competencias se tratan de desarrollar a través de las prácticas de aula: las transversales a través de actividades colaborativas en las propias sesiones de prácticas y las técnicas tanto a través de los contenidos proporcionados como del trabajo autónomo planteado en forma de trabajos individuales.

\section{Metodología y plan de trabajo}

- Para el objetivo 1: Planear trabajos individuales que el alumno pueda realizar como trabajo autónomo durante el curso en el contexto del aprendizaje basado en problemas e incorporar su evaluación como parte de la calificación final de la asignatura.

- Para el objetivo 2: Orientar el trabajo autónomo hacia la potenciación de las competencias, tanto transversales como técnicas, necesarias para el desarrollo del trabajo en equipo de la asignatura.

- Para el objetivo 3: Plantear actividades participativas en las prácticas de aula que resulten amenas para el alumno y le aporten valor de cara a la evaluación de la asignatura.

- Para el objetivo 4: Concienciar al alumno de la importancia de las actividades desarrolladas como parte de las prácticas de aula y de su aprovechamiento durante el desarrollo del trabajo en equipo.

Las tareas propuestas dentro del plan de trabajo son las siguientes:

1. Planificación de la temática de las 7 sesiones de 2 horas de prácticas de aula.

2. Planificación de las actividades para cada sesión de prácticas de aula.

3. Planificación del trabajo autónomo planteado.

4. Planificación de la evaluación del trabajo autónomo.

5. Análisis del nivel de asistencia del alumnado a las sesiones de prácticas de aula. 
6. Análisis del rendimiento del trabajo autónomo del alumnado.

7. Análisis de la influencia del trabajo autónomo planteado sobre el trabajo en equipo.

8. Análisis de la valoración del alumnado mediante encuesta.

Las tareas 1-4 corresponden al diseño de las prácticas de aula, mientras que las tareas 5-8 corresponden al análisis de los resultados alcanzados durante el curso académico 2019-20.

\section{Diseño de las prácticas de aula}

Tarea 1: Temáticas planteadas para las prácticas de aula (PA1-PA7)

PA1: Cooperación en equipo; PA2: Desarrollo de servicio multimedia como trabajo en equipo: PA3: Diseño del servicio multimedia e interactivo; PA4: Front-end del servicio; PA5: Back-end del servicio; PA6: Streaming ; PA7: Real Time Communication (RTC).

Tarea 2: Actividades planteadas para cada sesión de las prácticas de aula

PA1: Cooperación en equipo

1. Lectura, discusión y resumen de documentación sobre aprendizaje cooperativo mediante trabajo en equipos de $3 / 4$ alumnos/as y técnica de puzzle (Aronson, 1978).

2. Realización de test de personalidad por parte del alumnado mediante la herramienta libre EduTeams ${ }^{1}$ del CSIC (Roig, 2019) para formación automática de equipos equilibrados.

PA2: Desarrollo de servicio como trabajo en equipo

1. Presentación de la herramienta colaborativa Microsof Teams ${ }^{2}$, a utilizar para el desarrollo del trabajo en equipo y práctica con la misma.

2. Presentación al alumnado de los requisitos funcionales del servicio a desarrollar como trabajo en equipo de la asignatura y los criterios de evaluación del mismo.

\section{PA3-7}

1. Presentación de los contenidos técnicos relacionados con la temática de la sesión.

2. Trabajo del alumnado a partir de los ejemplos y guiones incluidos.

Tarea 3: Trabajo autónomo planteado para cada sesión de las prácticas de aula

En las cinco últimas sesiones se plantean trabajos individuales voluntarios consistentes en la elaboración de vídeos de 5 minutos de duración máxima sobre la ejecución de código

\footnotetext{
${ }^{1}$ https://eduteams.iiia.csic.e

2 https://www.microsoft.com/teams
} 
(software) relativo a las sesiones. La entrega de los trabajos individuales se realizó a través de actividades de tipo taller del campus virtual de la universidad (plataforma LMS tipo Moodle). La información suministrada incluye: 1) Título descriptivo; 2) Breve descripción del contenido; 3) URL correspondiente al vídeo, previamente subido a una plataforma online; 4) Herramientas utilizadas y pasos seguidos para la elaboración del vídeo; 5) Referencias (URLs) consultadas.

Tarea 4: Evaluación del trabajo autónomo

Como corresponde a las actividades de tipo taller ${ }^{3}$, los trabajos fueron evaluados por pares entre el alumnado a través de una sencilla rúbrica, pudiendo además realimentar con comentarios a los autores de los trabajos. Se configuró cada taller de modo que un máximo de 5 alumnos revisaran el trabajo de cada autor, otorgando calificaciones entre 0 y 10 . La rúbrica proporcionada a los alumnos consta de 3 criterios con 5 niveles de valoración cada uno de ellos (Muy alta/Alta/Media/Baja/Muy baja): 1) Calidad de vídeo y audio; 2) Complejidad y utilidad en el contexto de la temática de la sesión; 3) Calidad y detalle de las explicaciones. Cada trabajo individual aporta hasta 0,4 puntos a la calificación final de la asignatura, siendo la máxima aportación del conjunto de trabajos 1 punto (frente a 3 puntos del trabajo en equipo, 3 puntos de las prácticas de laboratorio y 3 puntos de la teoría).

\section{Resultados}

Tarea 5: Asistencia a las sesiones de prácticas de aula

En la figura 1 se muestra el nivel de asistencia comparada de los dos últimos cursos, apreciándose un aumento significativo y sostenido en el curso actual (2019-2020). En el curso 2018-2019 no se contabilizó la asistencia a la segunda sesión y las dos últimas sesiones del curso actual no tuvieron lugar debido a la crisis sanitaria provocada por la pandemia de covid-19. La asistencia media durante las cinco sesiones del curso actual fue del $86 \%$.

Tarea 6: Rendimiento del trabajo autónomo

De un total de 42 alumnos presentados a las actividades de evaluación continua de la asignatura, 24 (57\%) realizaron alguno de los trabajos individuales propuestos. La calificación media de los trabajos fue de 8,51 puntos sobre 10 .

3 https://docs.moodle.org/all/es/Actividad_de_taller 


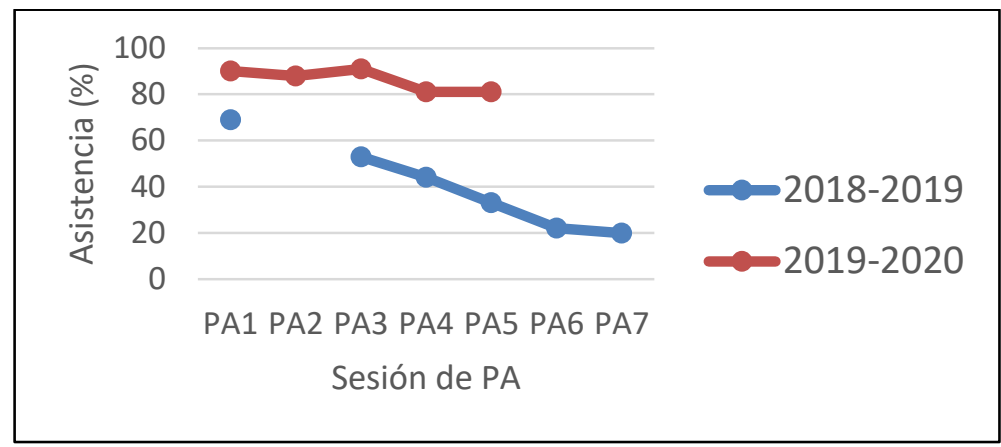

Fig. 1 Asistencia a las prácticas de aula

Tarea 7: Influencia del trabajo autónomo sobre el rendimiento en el trabajo en equipo

Uno de los objetivos más importantes del proyecto es tratar de comprobar si el aprovechamiento del trabajo autónomo planteado en las nuevas prácticas de aula en forma de trabajos individuales ha podido influir sobre los resultados del trabajo en equipo.

Tabla 1. Desglose de la calificación individual del trabajo en equipo

\begin{tabular}{|c|c|c|}
\hline Bloque & Item & Puntos \\
\hline \multirow{3}{*}{$\begin{array}{l}\text { Servicio desarrollado } \\
(60 \% \text {, común a todos los } \\
\text { miembros })\end{array}$} & Funcionalidad & 4 \\
\hline & Memoria & 1,5 \\
\hline & Valoración del resto de equipos & 0,5 \\
\hline \multirow{2}{*}{$\begin{array}{l}\text { Defensa del trabajo } \\
(20 \%, \text { individual) }\end{array}$} & Intermedia & 1 \\
\hline & Final & 1 \\
\hline \multirow{4}{*}{$\begin{array}{l}\text { Cooperación alcanzada } \\
(20 \% \text {, individual) }\end{array}$} & Actividad en el repositorio de software (Git) & 1 \\
\hline & Comunicación mediante Teams (Chat) & 0,5 \\
\hline & Actividad en reuniones del equipo & 0,5 \\
\hline & TOTAL & 10 \\
\hline
\end{tabular}

En la tabla 1 se muestra el desglose de la calificación del alumno en el trabajo en equipo. El $60 \%$ de dicha calificación es común a todos los miembros del equipo y el $40 \%$ restante individual. Comparando las calificaciones obtenidas en el curso actual con el anterior la calificación media ha pasado de 8,09 a 8,42, lo que supone una mejora del 5\%. Teniendo en cuenta que no todos los alumnos han realizado el trabajo autónomo planteado en las prácticas de aula, podemos establecer otra interesante comparativa entre las calificaciones medias en los trabajos en equipo de los alumnos que no han realizado (TEim_nTA) y sí han realizado (TEim_TA) dicho trabajo autónomo. Las calificaciones medias son de 8,17 y 8,6 respetivamente, lo que supone una mejora del 5,31\%. Además de la mejora observada, hay que tener en cuenta que el trabajo autónomo no solo influye en la calificación propia de un alumno en el trabajo en equipo, sino también en la de sus compañeros de equipo, hayan 
realizado o no trabajo autónomo, ya que recordemos que el $60 \%$ de la calificación del trabajo en equipo es común a todos los miembros.

A continuación, para aquellos alumnos que han realizado los trabajos individuales planteados, analizamos la relación entre las calificaciones del trabajo autónomo (TAi) y las calificaciones en los trabajos en equipo (TEi). En las calificaciones del trabajo autónomo se ha normalizado a 10 la puntuación total obtenida por cada alumno (2 puntos como máximo), aunque tan solo sea necesario un punto para alcanzar la máxima calificación en la asignatura.
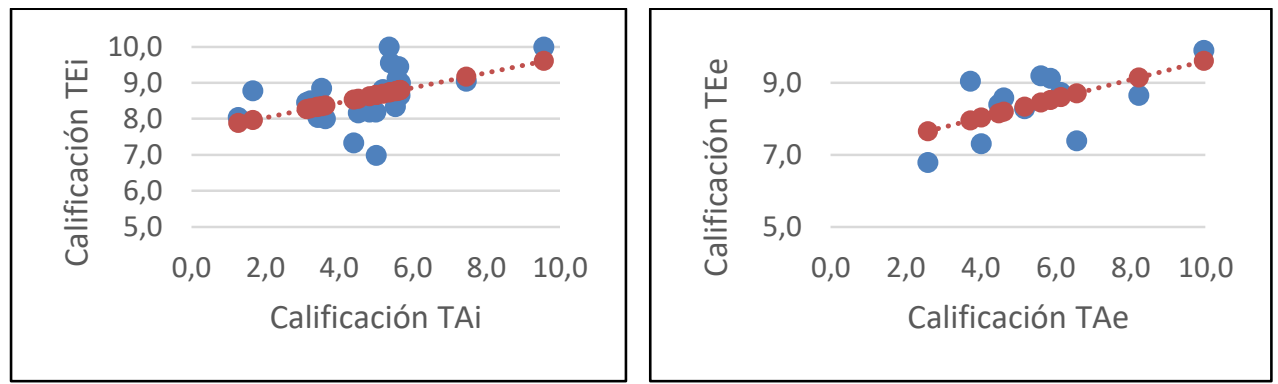

Fig. 2 Relación entre calificaciones de trabajos individuales/equipo

En la figura 2(izquierda) se muestra el diagrama de dispersión correspondiente a la comparativa de calificaciones y la aproximación lineal a la nube de puntos. El índice de correlación de Pearson es de 0,49, lo que supone una correlación media. Teniendo en cuenta lo que indicábamos anteriormente sobre la influencia del trabajo autónomo de cada alumno sobre el resto de los miembros de su equipo, se ha decidido comparar las calificaciones medias del trabajo autónomo de los miembros de cada equipo (TAe) con las correspondientes calificaciones medias del trabajo en equipo (TEe), con objeto de comprobar cómo evoluciona la correlación en este caso. Así, por ejemplo, en el caso de que solo un alumno haya realizado el trabajo autónomo en un equipo de 4 miembros, la calificación media resultante bajaría a la cuarta parte. En la figura 2(derecha) se muestra el nuevo diagrama de dispersión correspondiente a la relación así como la aproximación lineal a la nube de puntos. El índice de correlación de Pearson sube en este caso hasta 0,60, lo que supone una correlación media-alta. Aunque no se puede establecer una relación causal directa entre las calificaciones en el trabajo autónomo y en el trabajo en equipo, parece razonable pensar que sí existe una significativa influencia.

Tarea 8:Valoración por el alumnado

La valoración por parte del alumnado se ha medido con las 3 cuestiones indicadas en la tabla 2, planteadas a través de una encuesta online de respuesta anónima y voluntaria con una escala de valoración de Likert de 7 niveles. En la encuesta se obtuvieron 29 respuestas 
válidas, lo que implica un $69 \%$ del alumnado. Esta participación se considera suficientemente representativa. Como resultado estadístico de las valoraciones correspondientes a la primera pregunta se obtuvo una mediana de 5 y un valor medio de 4,8 .

\section{Tabla 2. Encuesta de satisfacción}

1) ¿Cómo valoras la estrategia de formación automática de los equipos a través de EduTeams desde tu experiencia con el trabajo en equipo

2) ¿Cómo valoras la contribución de las siguientes actividades a tu calificación en el trabajo en equipo?

a) Trabajo autónomo llevado a cabo a partir de los recursos proporcionados en las PAs

b) Trabajo autónomo llevado a cabo con la elaboración de los vídeos planteados como trabajos individuales en las PAs (contesta solo en el caso de que hayas realizado alguno)

c) Análisis de vídeos elaborados por otros miembros de tu equipo o de otros equipos

3) ¿Cómo valoras la calificación que has obtenido en cada parte de la asignatura y tu calificación final en relación al esfuerzo realizado?

a) Teoría; b) Prac. Laboratorio (PLs); c) Trabajo Equipo (TE); d) Prac. Aula (PAs); e) Calificación final (CF)
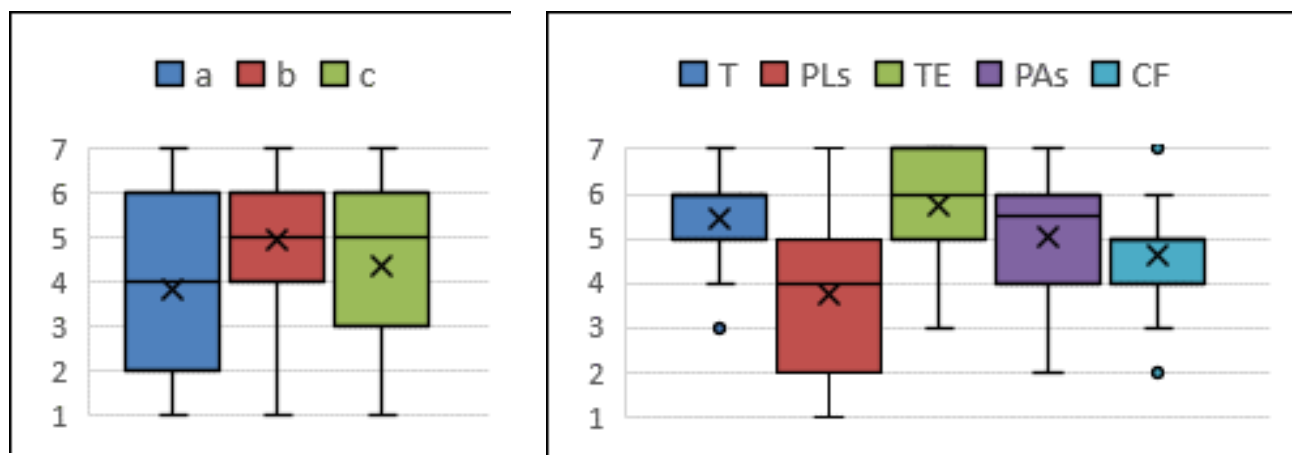

Fig. 3 Respuestas a la encuesta de satisfacción (preguntas 2 y 3)

El resultado de las valoraciones correspondientes a la segunda pregunta se resume en la figura 3(izquierda), donde se muestran cuartiles, mediana, valor medio (símbolo x) y valores atípicos (símbolo $O$ ) correspondientes a cada una de las tres distribuciones de valoraciones: a, b y c. Las valoraciones correspondientes a la pregunta $2 \mathrm{a}$ presentan una dispersión simétrica en todo el rango de valoración, estando situada la mediana justo en la mitad del rango (4). Ello divide en dos grupos iguales al alumnado, los que consideran una influencia media o alta del trabajo autónomo a partir de los contenidos de las PAs sobre la calificación del trabajo en equipo y los que consideran una influencia baja o muy baja. Entre estos últimos son mayoría los que no han realizado trabajos individuales, pero en todo 
caso podemos concluir que tres cuartas partes del alumnado consideran significativa la influencia. Respecto a las valoraciones correspondientes a las preguntas $2 \mathrm{~b}$ y $2 \mathrm{c}$, sus distribuciones son muy similares, con una mediana de 5, lo que denota una influencia significativamente mayor percibida por el alumnado. Esto pone de manifiesto la importancia de haber realizado los trabajos individuales y haber analizado los de otros compañeros y compañeras.

El resultado de las valoraciones correspondientes a la tercera pregunta se resume en la figura 3(derecha), donde se muestran las cinco distribuciones de valoraciones correspondientes a las partes de la asignatura. Las valoraciones correspondientes a las preguntas 3c (TE) y 3d (PAs) son las que más interesan en el marco de este estudio, aportando las otras tres interesante información contextual. Las valoraciones sobre la calificación final en el trabajo en equipo en relación con el esfuerzo realizado son las más elevadas, con una mediana de 6 , y ello pese al gran esfuerzo temporal reportado por el alumnado. En cuanto a las valoraciones sobre la calificación final en las prácticas de aula en relación con el esfuerzo realizado se sitúan en tercera posición, con una mediana de 5,5 y con tres cuartas partes del alumnado indicando valoración media o alta.

\section{Conclusiones}

El nuevo diseño de las prácticas de aula ha demostrado una influencia positiva sobre la motivación del alumnado para asistir a las sesiones presenciales. Ha mejorado el rendimiento de los trabajos en equipo y se ha verificado una significativa correlación entre el rendimiento en el trabajo autónomo planteado y el rendimiento en el trabajo en equipo, confirmado además con la encuesta a los alumnos. Aunque no todos los miembros de los equipos han llevado a cabo el trabajo autónomo, el realizado por alguno repercute sobre los resultados del equipo. Todo lo anterior se ha conseguido además con una satisfacción razonable del alumnado. No se ha conseguido en cambio motivar a todos los alumnos para abordar el trabajo autónomo volunario planteado en las prácticas de aula. Un reto para el próximo curso será aumentar el ratio de alumnos que lleven a cabo el trabajo autónomo.

\section{Referencias}

Anaya, A.R. and Boticario, J.G. (2011). Application of machine learning techniques to analyse student interactions and improve the collaboration process. Expert Systems with Applications, Elsevier, 38, 1171-1181. 
Ardid, M. et Al. (2014). Las prácticas de aula y el trabajo en equipo: una oportunidad para que los alumnos adquieran dimensiones competenciales. Actas del XXII Congreso Universitario de Innovación Educativa en las Enseñanzas Técnicas (CUIEET), Almadén, 1443-1444.

Aronson, E., et al. (1978). The jigsaw classroom. Sage.

Burke, A. (2011). Group Work: How to Use Groups Effectively. Journal of Effective Teaching, 11(2), $87-95$.

Lingard, R. and Barkataki, S. (2011). Teaching Teamwork in Engineering and Computer Science. Proceedings of the ASEE/IEEE Frontiers in Education Conference (Session TIA 1-5), Rapid City.

Marin-Garcia, J. A. and Lloret, J. (2008). Improving Teamwork with University Engineering Students. The Effect of an Assessment Method to Prevent Shirking. WSEAS Transactions on Advances in Engineering Education, 5(1), 1790-1979.

Monterrey, D. (2008). Las estrategias y técnicas didácticas en el rediseño: el aprendizaje basado en problemas como técnica didáctica. Instituto Tecnológico y de Estudios Superiores de Monterrey.

Perera, D., Kay, J., Koprinska, I., Yacef, K. and Zaïane, O.R. (2009). Clustering and Sequential Pattern Mining of Online Collaborative Learning Data. IEEE Transactions on Knowledge and Data Engineering, 21(6), 759-772.

Roig, C. et Al. (2019). Diverse Teams in the Classroom. International Conerence on AI + Adaptive Education (AIAED19), Beijing.

Suárez, F. J. et Al. (2017). Alumnos más Participativos con el uso de Herramientas de Gamificación y Cooperación. Actas del XXVI Congreso Universitario de Innovación Educativa en las Enseñanzas Técnicas (CUIEET), Gijón, 985-996.

Viswanathan, S. A. and VanLehn, K. (2017). Using the tablet gestures and speech of pairs of students to classify their collaboration. IEEE Transactions on Learning Technologies, 11(2), 230-242. 